\title{
Modelagem do rendimento no desdobro de toras de Manilkara spp. (Sapotaceae) em serraria do estado de Roraima, Brasil
}

\author{
Yield modeling in sawing logs of Manilkara spp. \\ (Sapotaceae) in sawmill in the state of Roraima, Brazil
}

\author{
Filipe Eduardo Danielli ${ }^{1}$, Bruno Oliva Gimenez ${ }^{1}$, Criscian Kellen Amaro de Oliveira ${ }^{1}$, \\ Joaquim dos Santos ${ }^{2}$ e Niro Higuchi ${ }^{3}$
}

\begin{abstract}
Resumo
O objetivo desse estudo foi estimar o rendimento no desdobro de toras de Manilkara spp., quantificar os subprodutos, avaliar diferença de rendimento entre as classes diamétricas e ajuste de modelos para estimativa do rendimento em madeira serrada e estimativa do volume da parte ocada das toras. Foram amostradas 71 toras, agrupadas em classes de diâmetro. Foi determinado o volume das toras pelo método de Smalian e calculado o volume de madeira serrada, para determinação do rendimento. Foram testados 12 modelos para estimativa do rendimento em madeira serrada e 12 modelos para estimativa do volume do oco das toras. A escolha dos melhores modelos foi feita baseando-se no maior coeficiente de determinação ajustado $\left(\mathrm{R}_{\text {ajust }}^{2}\right)$, menor erro padrão da estimativa $\left(\mathrm{S}_{\mathrm{yx}} \%\right)$ e distribuição homogênea dos resíduos. $\mathrm{O}$ rendimento médio foi de $30,1 \%$ e não houve diferença estatística no rendimento entre as classes diamétricas e entre toras ocadas e não ocadas. A classe $(70<79,9 \mathrm{~cm})$ foi a que apresentou o melhor rendimento. Para estimativa de rendimento, a melhor foi a equação 7. Para estimativa do volume do oco, as melhores foram as equações dois, três e dez.
\end{abstract}

Palavras-chave: Serraria, maçaranduba, processamento de toras, madeira serrada, subprodutos.

\begin{abstract}
The aim of this study was to estimate the yield of sawing Manilkara spp. logs, to quantify the wood by products generated, to evaluate differences in the yield between the diameter classes and to adjust models to estimate the yield in lumber and to estimate the volume of the hollow part of the logs. Seventy-one logs were sampled and grouped into diameter classes. Log volumes were determined by the Smalian method and the volume of lumber was calculated to determine the yield. Twelve models were tested to estimate the sawn lumber and twelve models to estimate the volume of the hollow logs. The choice of the best models was made based on the highest adjusted coefficient of determination $\left(R^{2}{ }_{\text {ajust }}\right)$, lowest standard error of estimate (Syx\%) and homogeneous distribution of the residues. The average yield was $30.1 \%$ and showed no statistical differences in yield between the diameter classes and between hollow logs and nonhollow logs. Class $5(70<79,9 \mathrm{~cm})$ was the one that presented the best yield. To estimate the yield, the best equation was the number seven. To estimate the volume of the hollow part of the logs, the best equations were number two, three and ten.
\end{abstract}

Keywords: Sawmill, maçaranduba, log processing, sawn wood, wood by-products.

\section{INTRODUÇÃO}

A Amazônia brasileira é uma das maiores regiões produtoras de madeira tropical do mundo, perdendo apenas para a Indonésia e Malásia. Com o esgotamento dos estoques de madeira desses países, o Brasil ganharia cada vez mais destaque no mercado internacional como principal fornecedor para suprir essa demanda (CLEMENT; HIGUCHI, 2006).

As serrarias são empresas responsáveis pelo desdobro primário da madeira em tora em madeira serrada. Apresentam baixo rendimento industrial em função do nível tecnológico obsoleto envolvi-

\footnotetext{
${ }^{1}$ Mestrandos do Programa de Pós-Graduação em Ciências de Florestas Tropicais. INPA - Instituto Nacional de Pesquisas da Amazônia. Av. André Araújo, 2936 - Petrópolis, CEP: 69060-001 - Manaus - AM. Brasil. E-mail: fklauberg@hotmail.com; bruno.oliva.gimenez@gmail.com; crisciankellen@gmail.com.

${ }^{2}$ Pesquisador Doutor. INPA - Instituto Nacional de Pesquisas da Amazônia. Av. André Araújo, 2936 - Petrópolis, CEP: 69060-001 - Manaus - AM. Brasil. E-mail: joca@inpa.gov.br.

3Pesquisador Ph.D. INPA - Instituto Nacional de Pesquisas da Amazônia. Av. André Araújo, 2936 - Petrópolis, CEP: 69060-001 - Manaus - AM. Brasil. E-mail: niro@inpa.gov.br.
} 
do no processo de produção (BRAND et al., 2002). O que implica no aumento de custo do produto final e na geração de uma grande quantidade de resíduos (BIASI; ROCHA, 2002).

Na Amazônia, o rendimento médio da maioria das espécies processadas nas serrarias é de aproximadamente 30\% (CLEMENT; HIGUCHI, 2006), o que desperta atenção para a grande quantidade de subprodutos gerados.

O rendimento no desdobro das toras na serraria é uma importante questão que tem relação intrínseca com a sustentabilidade na utilização dos recursos florestais, uma vez que o nível de aproveitamento da matéria-prima influencia diretamente sobre a área de floresta explorada necessária para atender a demanda por madeira.

A presença de oco em algumas espécies amazônicas, como a maçaranduba, pode implicar na necessidade de um volume maior de madeira em toras para atender a uma certa demanda em madeira serrada, dificultando as estimativas de rendimento e produtividade.

Além disso, existem pesquisadores que acreditam ser inviável a exploração de toras ocadas, do ponto de vista econômico e ecológico. Porém, no caso da maçaranduba, em função da incidência de rachaduras e trincas na parte medular das toras, mesmo as toras não estando ocadas, a parte central não é aproveitada. Isso pode implicar no rendimento similar em madeira serrada de toras ocadas e de toras não ocadas.

A modelagem tem sido utilizada amplamente na área florestal para realizar previsões de produtividade florestal, estimativas hipsométricas, volumétricas e de biomassa (SANTOS, 1996; BARROS et al., 2002).

O uso de modelos para estimativa do rendimento de serrarias é importante para os empresários do setor florestal, pois facilitam o prognóstico do estoque e rendimento da matéria-prima processada na serraria, viabilizando a melhoria no planejamento, otimização e controle na produção (IWAKIRI, 1990).

Contudo, apesar de reconhecida a importância do uso de modelos matemáticos para estimativa de rendimento de espécies madeireiras em serrarias, eles ainda têm sido pouco utilizados no Brasil (ABREU, 2000; SOUZA et al., 2007; VALÉRIO et al., 2009).

Assim, fica evidente a necessidade de pesquisas que disponibilizem informações técnicas sobre o rendimento de espécies tropicais desdobradas nas serrarias. Principalmente as com maior demanda no mercado, como a maçaranduba (Manilkara spp., Sapotaceae), que é uma das espécies mais abundantes na Amazônia (HIRAI et al., 2008) e uma das mais exploradas pelos madeireiros, em função de sua coloração escura e dureza, sendo utilizada em quase $90 \%$ das serrarias da região amazônica (RIBEIRO, 2008).

Neste contexto, o propósito deste trabalho foi estimar o rendimento no processo de desdobro de toras de Manilkara spp. e ajustar modelos para estimativa do rendimento em madeira serrada e estimativa do volume da parte ocada das toras.

\section{MATERIAL E MÉTODOS}

Este estudo foi desenvolvido em uma serraria situada no município de Rorainópolis, sul do estado de Roraima, distante cerca de $300 \mathrm{~km}$ de Boa Vista-RR. A serraria onde foi desenvolvido o estudo é representativa das demais que processam madeira na Amazônia. A figura 1 mostra o fluxograma da linha de produção da serraria.

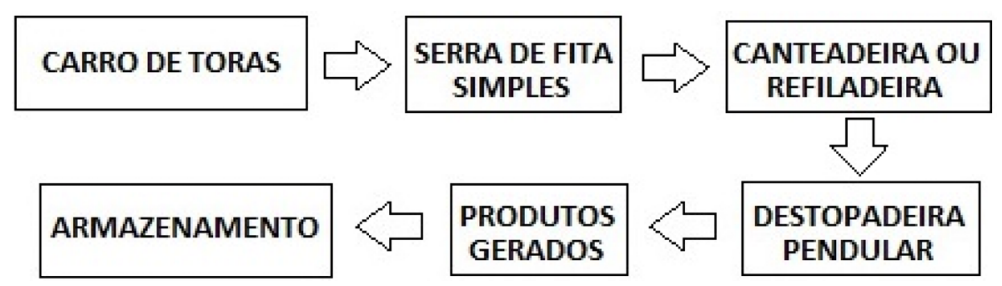

Figura 1. Fluxograma das etapas do processo de desdobro na serraria.

Figure 1. Flowchart of the steps of the sawing process of the logs in sawmill.

Foram selecionadas 71 toras aleatoriamente no pátio da empresa, sendo 40 toras não-ocadas e 31 toras ocadas. Em seguida, as toras foram marcadas e cada uma recebeu um número. Após a 
marcação, levando em consideração o diâmetro da ponta fina $\left(\mathrm{d}_{\mathrm{pf}}\right)$, as toras foram agrupadas em classes diamétricas (tabela 1), aplicando-se a fórmula de Sturges (ANGELINI; MILONE, 1993), muito utilizada para definição da quantidade de número de classes para um conjunto de dados. A fórmula 1 é dada por:

Onde:

$$
N C=1+1,4427 \operatorname{Ln}(n)
$$

$\mathrm{NC}=$ Número de classes

$\mathrm{Ln}=$ Logaritmo natural

$\mathrm{n}=$ Número de observações (toras)

Tabela 1. Quantidade, diâmetro médio e comprimento médio das toras agrupadas por classes de diâmetro. Table 1. Quantity, average diameter and average length of the logs grouped by diameter classes.

\begin{tabular}{lcccc}
\hline Classes & $\begin{array}{c}\text { Intervalo de diâmetro na } \\
\text { ponta fina da tora }(\mathbf{c m})\end{array}$ & $\begin{array}{c}\text { Número } \\
\text { de toras } \mathbf{( n )}\end{array}$ & $\begin{array}{c}\text { Diâmetro } \\
\text { médio }(\mathbf{c m})\end{array}$ & $\begin{array}{c}\text { Comprimento } \\
\text { médio }(\mathbf{m})\end{array}$ \\
\hline 1 & $30<39,9$ & 9 & 36,26 & 5,11 \\
2 & $40<49,9$ & 15 & 44,08 & 7,10 \\
3 & $50<59,9$ & 21 & 55,12 & 6,76 \\
4 & $60<69,9$ & 13 & 63,79 & 7,62 \\
5 & $70<79,9$ & 7 & 74,18 & 6,64 \\
6 & $>80$ & 6 & 86,88 & 6,67 \\
\hline
\end{tabular}

Para calcular o volume de cada tora com casca foi feita a marcação das seções, a cada metro e, com o auxílio da suta diamétrica, foi realizada a coleta de duas medidas de diâmetro, no início e fim de cada seção, sendo calculada a média para cada uma. Foi medido o diâmetro da ponta fina $\left(\mathrm{d}_{\mathrm{pf}}\right)$ de cada tora, utilizado como variável de entrada para o ajuste dos modelos de rendimento.

Foi feita a cubagem rigorosa de cada tora, utilizando-se o método de Smalian (MACHADO; FIGUEIREDO FILHO, 2006) pela fórmula 2:

$$
V t=\left(\frac{\left(\frac{\pi * \mathrm{D}_{1}^{2}}{40000}\right)+\left(\frac{\pi * \mathrm{D}_{2}^{2}}{40000}\right)}{2}\right) * \mathrm{~L}+\cdots+\left(\frac{\left(\frac{\pi * \mathrm{D}_{\mathrm{n}}^{2}}{40000}\right)+\left(\frac{\pi * \mathrm{D}_{n}^{2}}{40000}\right)}{2}\right) * \mathrm{~L}
$$

Onde:

$\mathrm{Vt}=$ volume da tora com casca $\left(\mathrm{m}^{3}\right) ; \mathrm{D}_{1}=$ diâmetro da seção $1(\mathrm{~cm}) ;$

$\mathrm{D}_{2}=$ diâmetro da seção $2(\mathrm{~cm}) ; \mathrm{D}_{\mathrm{n}}=$ diâmetro da seção $\mathrm{n}(\mathrm{cm})$;

$\mathrm{L}=$ comprimento da seção $(\mathrm{m})$;

A cubagem para o cálculo do volume das toras ocas foi realizada normalmente pelo método de Smalian sem descontar o oco, de acordo com a fórmula anteriormente descrita. No entanto, foi necessária a cubagem do volume do oco (Vo), usado para o ajuste dos modelos matemáticos.

Para isso, com o auxílio de trena métrica, foi mensurado o comprimento do oco da tora (que se estendeu de uma extremidade a outra de todas as toras com oco) e calculada a média de duas medidas perpendiculares do diâmetro do oco no início $\left(D_{i}\right)$ e no final $\left(D_{f}\right)$ de cada extremidade da tora, conforme ilustrado na figura 2.

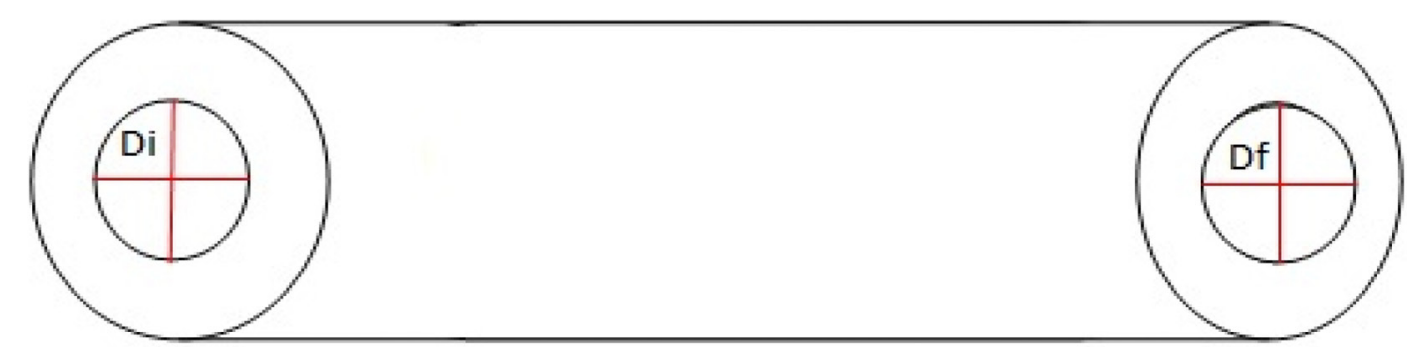

Figura 2. Medição do diâmetro médio no início e no fim de cada extremidade da tora para cubagem do volume da parte ocada.

Figure 2. Measurement of the average diameter at the beginning and at the end of each extremity log to determine the volume of the hollow part. 
Sendo:

$$
\begin{aligned}
& D_{i}=\frac{D_{i}^{\prime}+D_{i}^{\prime \prime}}{2} \\
& D_{f}=\frac{D_{f}^{\prime}+D_{f}^{\prime \prime}}{2}
\end{aligned}
$$

Em seguida foi realizada a mensuração do volume da parte ocada da tora por meio da fórmula 5:

$$
V_{o}=\frac{\left(\frac{\pi * D_{i}^{2}}{40000}\right)+\left(\frac{\pi * D_{f}^{2}}{40000}\right)}{2} * L
$$

Onde:

$\mathrm{V}_{\mathrm{o}}=$ volume do oco $\left(\mathrm{m}^{3}\right)$;

$\mathrm{D}_{\mathrm{i}}=$ diâmetro médio do oco da ponta fina da tora $(\mathrm{cm})$;

$\mathrm{D}_{\mathrm{f}}=$ diâmetro médio do oco da ponta grossa da tora $(\mathrm{cm})$;

$\mathrm{L}=$ Comprimento do oco $(\mathrm{m})$;

Após o desdobro, todas as peças geradas de cada tora foram separadas e cada peça teve suas dimensões medidas, com auxílio de paquímetro digital e trena métrica, para o cálculo do volume individual, utilizando a fórmula 6 :

$$
V m s_{i}=\frac{a * b * c}{10^{6}}
$$

Onde:

$\mathrm{Vms}_{\mathrm{i}}=$ volume individual da peça $\left(\mathrm{m}^{3}\right) ; \mathrm{a}=$ largura da peça $(\mathrm{cm})$; $\mathrm{b}=$ espessura da peça $(\mathrm{cm}) ; \mathrm{c}=$ comprimento da peça $(\mathrm{m})$;

Em seguida, foi calculado o volume de madeira serrada de cada tora, somando o volume das peças, utilizando a fórmula 7 :

Onde:

$$
V m s=\sum V m s_{i}
$$

Vms = volume em madeira serrada $\left(\mathrm{m}^{3}\right)$;

$\Sigma \mathrm{Vms}_{\mathrm{i}}=$ somatório do volume individual das peças $\left(\mathrm{m}^{3}\right)$;

A determinação do rendimento no desdobro das toras foi realizada por meio da razão entre o volume em madeira serrada e o volume com casca das toras processadas, dado pela fórmula 8 (BATISTA; CARVALHO, 2007; GOMIDE, 1974):

Onde:

$$
R(\%)=\frac{V_{m s}}{V_{t}} * 100
$$

$\mathrm{R}(\%)=$ rendimento percentual;

$\mathrm{V}_{\mathrm{ms}}=$ volume em madeira serrada $\left(\mathrm{m}^{3}\right)$;

$\mathrm{Vt}=$ volume com casca da tora $\left(\mathrm{m}^{3}\right)$;

Para verificar se havia diferença estatística do rendimento (\%) entre as classes diamétricas, foi feita uma análise de variância (ANOVA) com número de repetições diferente para cada classe de diâmetro (tabela 10), caracterizando dados desbalanceados, conforme metodologia utilizada por Dobner Jr. et al. (2012).

Foi utilizado o teste Tukey a 95\% de probabilidade para discriminar as diferenças entre as médias dos tratamentos. Foram utilizadas letras iguais para as médias que não apresentaram diferença estatística entre si e letras diferentes para as médias que apresentaram diferença estatística. Foi realizado o teste $t$ de Student a 95\% de probabilidade para verificar se havia diferença estatística do rendimento $(\%)$ entre toras ocadas e não ocadas. 
As análises estatísticas foram feitas com a utilização do software Excel 2007 e dos programas estatísticos Minitab 16 e Systat 12. Por meio de análise de regressão utilizando o método dos mínimos quadrados, foram testados 12 modelos para estimativa do rendimento em madeira serrada (tabela 2) e 12 modelos para estimativa do volume do oco (tabela 3 ).

Tabela 2. Modelos testados para estimar o rendimento de madeira serrada no desdobro de toras de Manilkara spp. Table 2. Models tested to estimate the yield of lumber in Manilkara spp. logs.

\begin{tabular}{lll}
\hline Número & Modelo & Fonte \\
\hline 1 & $R=B_{0}+B_{1}{ }^{*} D_{p f}+\varepsilon$ & - \\
2 & $R=B_{0}+B_{1}{ }^{*} D_{p f}+\varepsilon$ & - \\
3 & $R=B_{0}+B_{1}{ }^{*} D_{p f}+B_{2}{ }^{*} L+\varepsilon$ & Valério et al.(2009) \\
4 & $R=B_{0}+B_{1}{ }^{*} D_{p f}+B_{2}{ }^{*} L^{2}+\varepsilon$ & - \\
5 & $R=B_{0}+B_{1}{ }^{*}\left(D_{p f}{ }^{2 *} L\right)+\varepsilon$ & - \\
6 & $R=B_{0}{ }^{*} D_{p f}{ }^{B 1}+\varepsilon$ & Valério et al.(2009) \\
7 & $R=B_{0}{ }^{*} D_{p f}{ }^{B 1} L^{B 2}+\varepsilon$ & Valério et al.(2009) \\
8 & $R=B_{0}{ }^{*}\left(D_{p f}{ }^{*} L\right)^{B 1}+\varepsilon$ & - \\
9 & $R=B_{0}{ }^{*} D_{p f}{ }^{B 1} V t^{B 2}+\varepsilon$ & - \\
10 & $R=B_{0}{ }^{*} V t^{B 1}+\varepsilon$ & - \\
11 & $R=B_{0}+B_{1}{ }^{*} V t+\varepsilon$ & Valério et al.(2009) \\
12 & $R=B_{0}+B_{1}{ }^{*} V t+B_{2}{ }^{*} D_{p f}+\varepsilon$ & -
\end{tabular}

R: rendimento $\left(\mathrm{m}^{3}\right)$; $\mathrm{D}_{\mathrm{pf}}$; diâmetro da ponta fina da tora $(\mathrm{cm})$; L: comprimento da tora $(\mathrm{m})$; Vt: volume da tora com casca $\left(\mathrm{m}^{3}\right) ; \mathrm{B}_{0}, \mathrm{~B}_{1}$ e $\mathrm{B}_{2}: \mathrm{coefi}$ cientes da regressão e e: erro aleatório.

Tabela 3. Modelos testados para estimar o volume da parte ocada das toras de Manilkara spp.

Table 3. Models tested to estimate the volume of the hollow part of Manilkara spp. logs.

\begin{tabular}{lll}
\hline Número & Modelo & Fonte \\
\hline 1 & $V_{o}=B_{0}+B_{1}{ }^{*} D_{p f}+\varepsilon$ & - \\
2 & $V_{o}=B_{0}+B_{1}{ }^{*} D_{o}+\varepsilon$ & - \\
3 & $V_{o}=B_{0}+B_{1}{ }^{*} D_{o}+B_{2}{ }^{*} L+\varepsilon$ & - \\
4 & $V_{o}=B_{0}+B_{1}{ }^{*} D_{p f}+B_{2}{ }^{*} D_{o}+\varepsilon$ & - \\
5 & $V_{o}=B_{0}+B_{1}{ }^{*} D_{p f}+B_{2}{ }^{*} D_{o}+B_{3}{ }^{*} L+\varepsilon$ & - \\
6 & $V_{o}=B_{0}+B_{1}{ }^{*}\left(D_{o}{ }^{*} L\right)+\varepsilon$ & - \\
7 & $V_{o}=B_{0}+B_{1}{ }^{*}\left(D_{o}{ }^{*} D_{p f}\right)+\varepsilon$ & - \\
8 & $V_{o}=B_{0}+B_{1}{ }^{*} V t+\varepsilon$ & - \\
9 & $V_{o}=B_{0}{ }^{*} V t^{B 1}+\varepsilon$ & - \\
10 & $V_{o}=B_{0}{ }^{*} D_{o}{ }^{B 1}+\varepsilon$ & - \\
11 & $V_{o}=B_{0}{ }^{*} D_{p f}{ }^{B}{ }^{*} D_{o}{ }^{B 2}+\varepsilon$ & - \\
12 & $V_{o}=B_{0}{ }^{*} D_{0}{ }^{B 1}{ }^{*}\left(D_{p f}{ }^{*} D_{0}\right)^{B 2}+\varepsilon$ & - \\
\hline
\end{tabular}

Vo: volume do oco da tora $\left(\mathrm{m}^{3}\right)$; $\mathrm{D}_{\mathrm{pf}}$; diâmetro da ponta fina da tora $(\mathrm{cm})$; Do=diâmetro do oco da tora $(\mathrm{cm})$; L: comprimento da tora $(\mathrm{m})$; Vt: volume da tora com casca $\left(m^{3}\right) ; B_{0}, B_{1}, B_{2}$ e $B_{3}$ : coeficientes da regressão e $\varepsilon$ : erro aleatório.

A seleção do melhor modelo foi feita levando em consideração critérios estatísticos que indicam a qualidade do modelo, com maiores valores de coeficiente de determinação $\left(\mathrm{R}^{2}{ }_{\text {just }}\right)$, menores valores de erro padrão da estimativa $\left(\mathrm{S}_{\mathrm{yx}}\right)$ e distribuição homogênea dos resíduos (BELSLEY et al., 1980; DRAPER; SMITH, 1966; SANTOS, 1996):

\section{Coeficiente de determinação ajustado $\left(\mathbf{R}_{\text {ajust }}{ }\right)$}

Onde:

$$
\mathrm{R}_{\mathrm{ajust}}^{2}=\mathrm{R}^{2}-\left(\frac{\mathrm{k}-1}{\mathrm{n}-\mathrm{k}}\right) *\left(1-\mathrm{R}^{2}\right)
$$

$\mathrm{R}^{2}=$ coeficiente de determinação; $\mathrm{k}=$ número de coeficientes;

$\mathrm{n}=$ número de observações (toras);

\section{Erro padrão da estimativa $\left(S_{y x}\right)$}

$$
\text { Syx }=\sqrt{\frac{\sum\left(\mathrm{y}-y_{\text {est }}\right)^{2}}{\mathrm{n}-\mathrm{k}}}
$$


Onde:

$\mathrm{y}=$ volume de madeira serrada observado da tora $\left(\mathrm{m}^{3}\right)$;

$\mathrm{y}_{\text {est }}=$ volume de madeira serrada estimado da tora $\left(\mathrm{m}^{3}\right)$;

$\mathrm{n}=$ número de toras amostradas;

$\mathrm{k}=$ número de coeficientes;

\section{Erro padrão da estimativa percentual $\left(S_{\mathrm{yx}} \%\right)$}

Onde:

$$
\operatorname{Syx}(\%)=\left(\frac{\operatorname{Syx}}{\mathrm{x}}\right) * 100
$$

$\mathrm{S}_{\mathrm{xy}}=$ erro padrão da estimativa $\left(\mathrm{m}^{3}\right) ; \mathrm{x}=$ média da população;

\section{Distribuição de resíduos}

Onde:

$$
\operatorname{Res}(\%)=\frac{(\text { Vest }- \text { Vobs })}{\text { Vobs }} * 100
$$

$\mathrm{V}_{\text {est }}=$ volume estimado de madeira serrada $\left(\mathrm{m}^{3}\right)$;

$\mathrm{V}_{\mathrm{obs}}=$ volume observado de madeira serrada $\left(\mathrm{m}^{3}\right)$;

\section{RESULTADOS E DISCUSSÃO}

Foram cubadas rigorosamente 71 toras de Manilkara spp., das quais 40 toras não ocadas e 31 ocadas. A tabela 4 apresenta os resultados para os rendimentos obtidos nas 6 classes diamétricas estudadas. O rendimento médio encontrado para o desdobro de toras foi de 30,1\%, com tendência de maiores rendimentos para as maiores classes de diâmetro.

Os resultados apresentados na tabela 4 mostram que não houve diferença significativa do rendimento entre as classes de diâmetro $(p=0,078)$. Outros autores encontraram o mesmo resultado com outras espécies amazônicas (BIASI; ROCHA, 2002; IWAKIRI, 1990).

Tabela 4. Resultados obtidos para o desdobro das toras nas seis classes diamétricas.

Table 4. Results obtained for sawing logs in six diameter classes.

\begin{tabular}{lccccc}
\hline Classes & $\begin{array}{c}\text { Intervalo de diâmetro } \\
\text { na ponta fina da tora } \mathbf{( c m})\end{array}$ & $\begin{array}{c}\text { Número de } \\
\text { toras }(\mathbf{n})\end{array}$ & $\begin{array}{c}\text { Volume de } \\
\text { toras }\left(\mathbf{m}^{3}\right)\end{array}$ & $\begin{array}{c}\text { Volume de } \\
\text { madeira serrada }\left(\mathbf{m}^{3}\right)\end{array}$ & $\begin{array}{c}\text { Rendimento médio } \\
(\%)\end{array}$ \\
\hline 1 & $30<39,9$ & 9 & 5,1304 & 0,9887 & $20,12 \mathrm{a}$ \\
2 & $40<49,9$ & 15 & 18,6245 & 5,6033 & $28,85 \mathrm{a}$ \\
3 & $50<59,9$ & 21 & 39,2983 & 12,4085 & $31,51 \mathrm{a}$ \\
4 & $60<69,9$ & 13 & 33,2833 & 11,0709 & $32,80 \mathrm{a}$ \\
5 & $70<79,9$ & 7 & 21,7521 & 7,7584 & $36,20 \mathrm{a}$ \\
6 & $>80$ & 6 & 23,9268 & 7,2341 & $30,13 \mathrm{a}$ \\
\hline
\end{tabular}

Médias seguidas de mesma letra não diferem estatisticamente entre si pelo teste de Tukey a $95 \%$ de probabilidade.

Mesmo não havendo diferença estatística, é possível observar que a classe 1 foi a que apresentou o menor rendimento entre as demais $(20,12 \%)$ e a classe 5 , o maior rendimento $(36,20 \%)$. Esta diferença de aproximadamente 16 pontos percentuais entre a classe 1 e classe 5 é considerada muito importante dentro de uma escala de produção, tanto na redução dos custos de produção (MARCHESAN, 2012), como na redução dos subprodutos gerados pela serraria.

SUDAM (1981), avaliando o rendimento no desdobro de toras de maçaranduba em serraria no estado do Pará, constatou a mesma tendência de aumento até uma classe máxima de rendimento $(65<75 \mathrm{~cm})$, próxima à classe máxima de rendimento encontrada neste estudo $(70<79 \mathrm{~cm})$.

No entanto, as toras com diâmetro superior a $80 \mathrm{~cm}$, apresentaram uma tendência de decréscimo no rendimento, que pode ter ocorrido em função de 75\% das toras amostradas na classe 6 estarem ocadas. Tonini e Ferreira (2004), também constataram diminuição no rendimento em classes diamétricas maiores, ocasionada pela ocorrência de rachaduras, nós, protuberâncias e podridões de cerne (oco) nas toras estudadas. 
O rendimento médio para o desdobro de toras de maçaranduba obtido neste estudo ficou abaixo da média de 37\% encontrada para as serrarias do estado de Roraima (PEREIRA et al., 2010) e de 36\% para as serrarias do estado do Pará (GERWING et al., 2000), porém similar ao rendimento médio de 30\%, para espécies amazônicas, apontado por Clement e Higuchi (2006).

O rendimento encontrado neste trabalho ficou abaixo dos resultados obtidos por outros autores: Dutra et al. (2005), 32,30\%; Nascimento et al. (2006), 36,50\%; Cavallet et al. (2010), 35,18\% e Marchesan (2012), 29,88\%, que avaliaram o rendimento de espécies tropicais em serraria e próximo aos valores encontrados por Kock (1976), 35\% e Brand et al. (2002), 34,87\%, para espécies de florestas plantadas.

Outros autores, ao estudar o rendimento médio da maçaranduba, encontraram valores ainda mais superiores àquele obtido neste estudo: SUDAM (1981), 50\%; Araujo (2003), 48,13\% e Nascimento et al. (2006), 41,2\%.

O baixo rendimento encontrado neste estudo $(30,1 \%)$ está diretamente relacionado à qualidade das toras que foram processadas na serraria, uma vez que algumas delas apresentaram defeitos como rachaduras radiais, fissuras e trincas na parte central da tora $(56,3 \%$ da toras), bem como podridões e oco $(43,7 \%$ da toras).

Na tabela 5 são apresentados os resultados encontrados para o rendimento das toras ocadas e não ocadas, onde é possível observar que não houve diferença estatística do rendimento entre ambas $(\mathrm{p}=0,168)$.

Tabela 5. Rendimento percentual obtido para as toras ocadas e não ocadas.

Table 5. Percent yield obtained for hollow logs and non-hollow logs.

\begin{tabular}{lc}
\hline Toras & Rendimento (\%) \\
\hline Ocadas & 28,07 a \\
Não-ocadas & $31,65 \mathrm{a}$ \\
\hline
\end{tabular}

Médias seguidas de mesma letra não diferem estatisticamente entre si pelo teste $t$ de Student a $95 \%$ de probabilidade.

De maneira geral, este comportamento similar do rendimento das toras ocadas e não ocadas ocorreu porque, durante o processo de desdobro na serraria, a parte central (cerne) da maioria das toras não ocadas não pôde ser aproveitada, em função da ocorrência de trincas e rachaduras radiais, sendo descartada como subproduto em forma de blocos quadrangulares.

Na tabela 6 são apresentadas as equações geradas para estimativa do rendimento de madeira serrada, com seus respectivos valores de coeficiente de determinação $\left(\mathrm{R}^{2}{ }_{\text {ajust }}\right)$, erro padrão da estimativa $\left(\mathrm{S}_{\mathrm{yx}}\right)$ em $\mathrm{m}^{3}$ e erro padrão da estimativa percentual $\left(\mathrm{S}_{\mathrm{yx}} \%\right)$.

Tabela 6. Equações geradas para estimar o rendimento de madeira serrada no desdobro de toras de Manilkara spp. Table 6. Equations generated to estimate the lumber yield in sawing of Manilkara spp. logs.

\begin{tabular}{|c|c|c|c|c|}
\hline $\mathbf{N}^{\circ}$ & Equação & $\mathbf{R}_{\text {ajust }}^{2}$ & $S_{y x}\left(m^{3}\right)$ & $\mathrm{S}_{\mathrm{yx}}(\%)$ \\
\hline 1 & $R=-0,627019144+0,022312987^{*} D_{p f}$ & 0,512 & 0,316 & 5,905 \\
\hline 2 & $R=0,036261+0,000176^{*} D_{p f}^{2}$ & 0,466 & 0,330 & 6,178 \\
\hline 3 & $R=-1,11392+0,020467^{*} D_{p f}+0,087458^{*} L$ & 0,592 & 0,289 & 5,398 \\
\hline 4 & $R=-0,29224886+0,000163139 * D_{p f}^{2}+0,007724131 * L^{2}$ & 0,585 & 0,291 & 5,444 \\
\hline 5 & $\mathrm{R}=0,003949+0,0000269025^{\star}\left(\mathrm{D}_{\mathrm{pf}}^{2 *} \mathrm{~L}\right)$ & 0,622 & 0,278 & 5,196 \\
\hline 6 & $R=0,0000000010 * D_{p f}^{2,475}$ & 0,753 & 0,387 & 7,240 \\
\hline 7 & $\mathrm{R}=0,00000001^{*} \mathrm{D}_{\mathrm{pf}}^{2,320 *} \mathrm{~L}^{1,111}$ & 0,809 & 0,297 & 5,550 \\
\hline 8 & $\mathrm{R}=0,0000001^{*}\left(\mathrm{D}_{\mathrm{pf}}^{2 *} \mathrm{~L}\right)^{1,427}$ & 0,799 & 0,349 & 6,527 \\
\hline 9 & $R=1,417^{*} D_{p f}^{-0,451 *} V^{1,472}$ & 0,852 & 0,276 & 5,165 \\
\hline 10 & $\mathrm{R}=0,312^{*} \mathrm{Vt}^{1,052}$ & 0,787 & 0,271 & 5,069 \\
\hline 11 & $R=-0,06237+0,348498 * V t$ & 0,644 & 0,270 & 5,048 \\
\hline 12 & $R=0,113159-0,00573^{*} V t+0,422683^{*} D_{n f}$ & 0,643 & 0,270 & 5,050 \\
\hline
\end{tabular}

R: rendimento $\left(\mathrm{m}^{3}\right)$; $\mathrm{D}_{\mathrm{pf}}$ : diâmetro da ponta fina da tora $(\mathrm{cm})$; L: comprimento da tora $(\mathrm{m})$; Vt: volume da tora com casca $\left(\mathrm{m}^{3}\right)$; $\mathrm{B}_{0}$, $\mathrm{B}_{1}$ e $\mathrm{B}_{2}$ : coeficientes da regressão e $\varepsilon$ : erro aleatório.

É possível observar que as equações não lineares resultantes dos modelos 6 a 10 foram as que apresentaram os melhores resultados em relação aos obtidos para os modelos lineares, sendo o modelo 9, o que apresentou os melhores resultados, porém apresentou forte correlação entre as 
variáveis independentes Dpf e Vt $(\mathrm{r}=0,927)$, indicando um problema de multicolinearidade entre elas, tendo o mesmo ocorrido para o modelo 12.

O modelo 7 tendeu a gerar estimativas mais confiáveis, em função de apresentar o menor erro padrão da estimativa $\left(S_{y x}=5,550 \%\right)$, alto coeficiente de determinação $\left(R^{2}=0,809\right)$, sendo o modelo mais recomendado nesse estudo para a estimativa do rendimento em madeira serrada no desdobro de toras de Manilkara spp. (Figura 3).

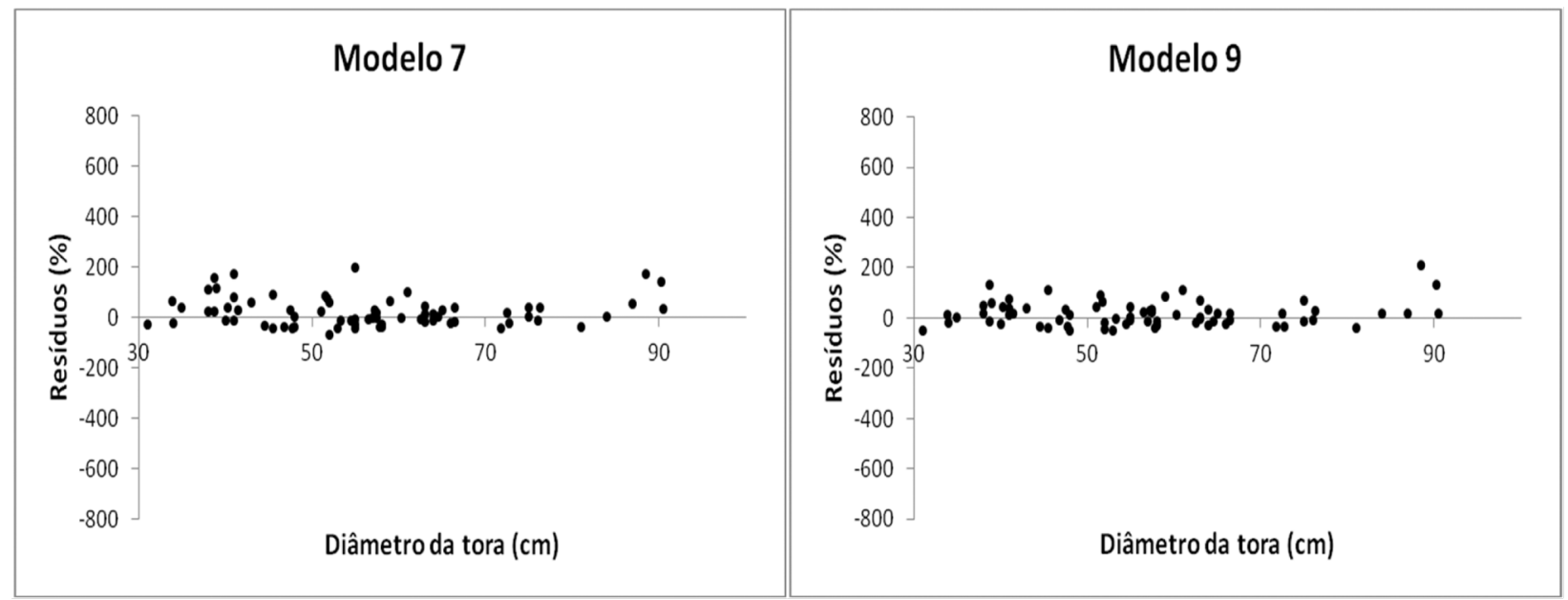

Figura 3. Distribuição gráfica dos resíduos dos modelos 7 e 9 testados para estimar o rendimento em madeira serrada. Figure 3. Graphic distribution of the residues of models 7 and 9 to estimate the lumber yield.

Na tabela 7 são apresentadas as equações geradas para estimativa do volume da parte ocada das toras, com seus respectivos valores de coeficiente de determinação $\left(\mathrm{R}_{\text {ajust }}^{2}\right)$, erro padrão da estimativa $\left(\mathrm{S}_{\mathrm{yx}}\right)$ em $\mathrm{m}^{3}$ e erro padrão da estimativa percentual $\left(\mathrm{S}_{\mathrm{yx}} \%\right)$.

Tabela 7. Equações geradas para estimar o volume do oco das toras de Manilkara spp.

Table 7. Equations generated to estimate the volume of the hollow of the Manilkara spp. logs.

\begin{tabular}{|c|c|c|c|c|}
\hline $\mathbf{N}^{\circ}$ & Equação & $\mathbf{R}_{\text {ajust }}^{2}$ & $\mathrm{~S}_{\mathrm{yx}}\left(\mathrm{m}^{3}\right)$ & $S_{y x}(\%)$ \\
\hline 1 & $V_{o}=-0,5640260395+0,0172255572 * D_{p f}$ & 0,305 & 0,344 & 11,499 \\
\hline 2 & $V_{o}=-0,4710134712+0,0343528950 * D_{0}$ & 0,911 & 0,123 & 4,108 \\
\hline 3 & $V_{o}=-1,04592316+0,03606338^{*} D_{0}+0,07310213^{*} L$ & 0,949 & 0,093 & 3,112 \\
\hline 4 & $V_{o}^{0}=-0,41301265-0,00138399^{*} D_{p f}^{0}+0,03539219^{*} D_{o}$ & 0,909 & 0,124 & 4,149 \\
\hline 5 & $V_{o}=-1,03364-0,00022 * D_{p f}+0,03622 * D_{o}+0,07276 * L$ & 0,947 & 0,095 & 3,168 \\
\hline 6 & $V_{0}=-0,5102458588+0,0050440069^{*}\left(D_{0}^{*} L\right)$ & 0,885 & 0,140 & 4,684 \\
\hline 7 & $V_{o}=-0,0842728476+0,0003150742 *\left(D_{0}^{*} D_{p f}\right)$ & 0,798 & 0,185 & 6,195 \\
\hline 8 & $V_{0}=-0,2591145330+0,3152790900^{*} \mathrm{Vt}$ & 0,425 & 0,313 & 10,458 \\
\hline 9 & $V_{0}=0,108^{*} V t^{1,658}$ & 0,469 & 0,306 & 10,225 \\
\hline & $V_{0}=0,001^{*} D_{0}^{1,728}$ & 0,916 & 0,122 & 4,063 \\
\hline & $V_{o}=0,005^{*} D_{p f}^{-0,557 *} D_{o}^{2,013}$ & 0,931 & 0,125 & 4,190 \\
\hline & $V_{o}=0,005^{*} D_{o}^{2,570 *}\left(D_{p f}^{*} D_{o}\right)^{-0,557}$ & 0,931 & 0,110 & 3,682 \\
\hline
\end{tabular}

Vo: volume do oco da tora $\left(\mathrm{m}^{3}\right)$; $\mathrm{D}_{\mathrm{pf}}$ : diâmetro da ponta fina da tora $(\mathrm{cm})$; Do=diâmetro do oco da tora $(\mathrm{cm})$; L: comprimento da tora $(\mathrm{m})$; Vt: volume da tora com casca $\left(m^{3}\right) ; B_{0}, B_{1}, B_{2}$ e $B_{3}$ : coeficientes da regressão e $\varepsilon$ : erro aleatório.

É possível observar que as equações resultantes dos modelos 2 a 5 (lineares) e 10 a 12 (nãolineares) foram as que obtiveram os melhores resultados estatísticos em relação aos demais.

Os modelos 3 e 5 tiveram desempenho similar, com o mesmo valor do coeficiente de determinação $\left(R_{\text {ajust }}^{2}=0,95\right)$ e baixos valores de erro padrão da estimativa, sendo o menor observado no modelo 3 (3,112\%), que apresentou uma melhor distribuição dos resíduos (Figura 4).

Os modelos 2 e 10 também são indicados para utilização, sendo recomendados por gerar estatísticas confiáveis para a estimativa do volume do oco das toras amostradas (Figura 5). Outra vantagem na utilização desses modelos em relação aos demais se dá em função de terem apenas o diâmetro do oco (Do) como variável independente, que representa maior facilidade no momento da coleta, com menor risco da incidência de erros amostrais (HIGUCHI et al., 1998). 


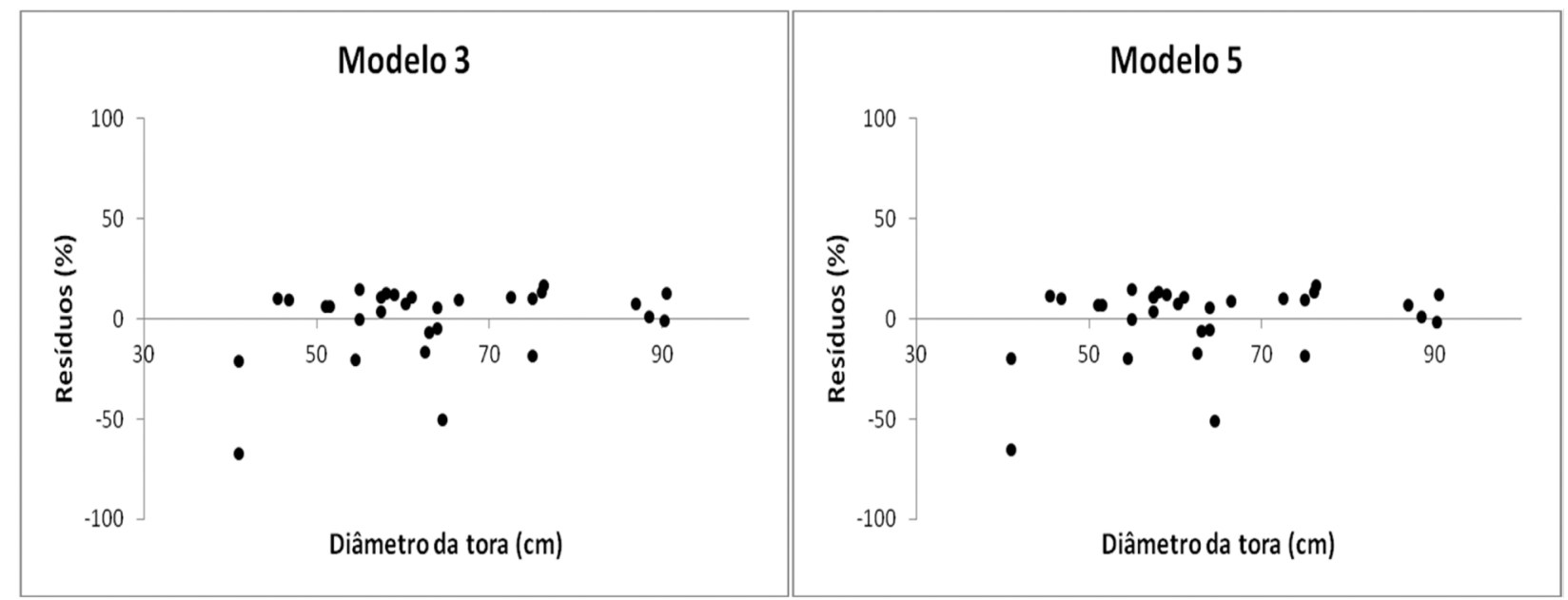

Figura 4. Distribuição gráfica dos resíduos dos modelos 3 e 5 testados para estimar o volume do oco das toras de Manilkara spp.

Figure 4. Graphic distribution of the residues of models 3 and 5 to estimate the volume of the hollow of the Manilkara spp. logs.

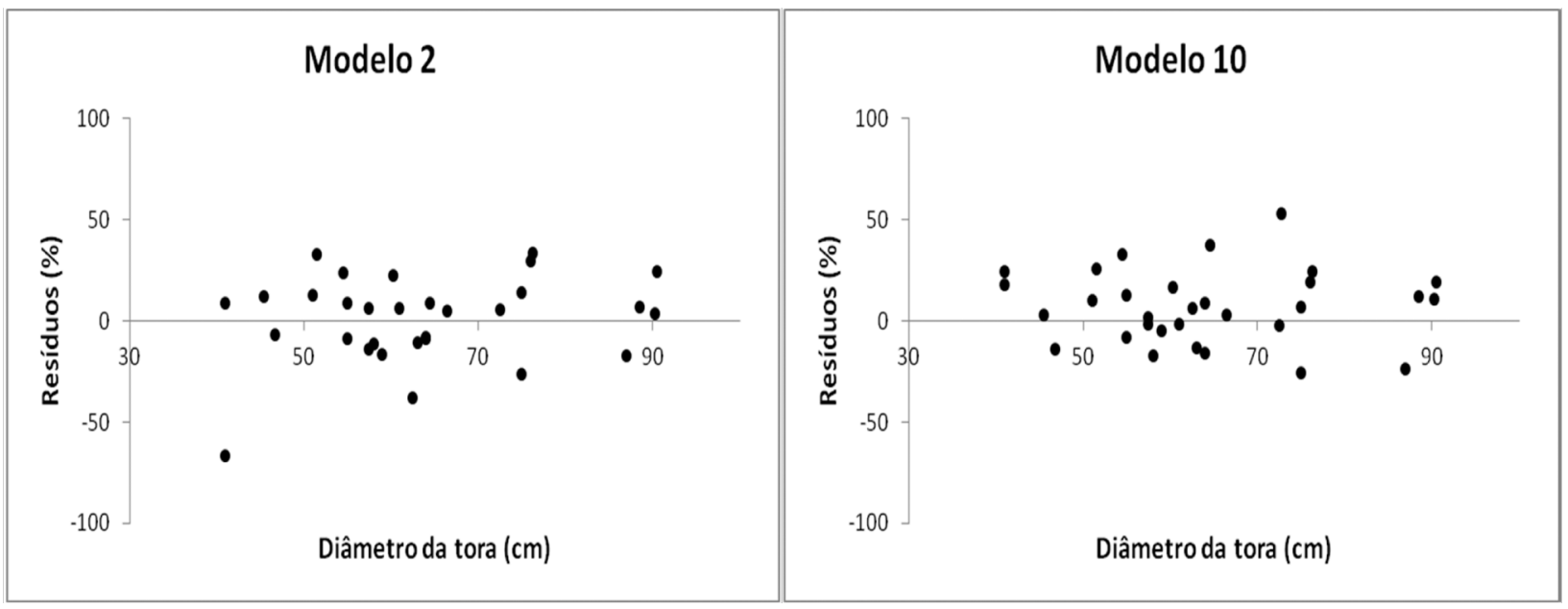

Figura 5. Distribuição gráfica dos resíduos dos modelos 2 e 10 testados para estimar o volume do oco das toras de Manilkara spp.

Figure 5. Graphic distribution of the residues of models 2 and 10 to estimate the volume of the hollow of the Manilkara spp. logs.

\section{CONCLUSÕES}

A maçaranduba (Manilkara spp.) apresentou baixo rendimento no desdobro de toras na serraria analisada com geração de subprodutos que não são comercializados.

Foi observado um aumento do rendimento com o incremento do diâmetro. Porém, na última classe diamétrica (classe 6), a tendência não foi a mesma, indicando decréscimo no rendimento, em função das toras de maiores diâmetros apresentarem defeitos internos e incidência de oco, com rendimento mínimo de 11,54\% e máximo de 59,60\%.

Este estudo mostrou não haver diferença significativa entre o rendimento em madeira serrada de toras ocadas e de toras não ocadas, bem como apontou também não ter havido diferença de rendimento entre as classes diamétricas analisadas.

A equação $7\left(\mathrm{R}=0,00000001 * \mathrm{Dpf}^{2,320 *} \mathrm{~L}^{1,111}\right)$ se mostrou mais adequada para estimativa do rendimento de madeira serrada de maneira confiável.

Aequação $2\left(V_{o}=-0,4710134712+0,0343528950 * D_{o}\right)$, equação3 $\left(V_{o}=-1,04592316+0,03606338 * D_{o}\right.$ $\left.+0,07310213{ }^{*} \mathrm{~L}\right)$ e equação $10\left(\mathrm{~V}_{\mathrm{o}}=0,001{ }^{*} \mathrm{D}_{\mathrm{o}}{ }^{1,728}\right)$ apresentaram índices estatísticos semelhantes e permitem estimar o volume da parte ocada das toras com maior confiabilidade.

$\mathrm{O}$ ajuste de modelos por meio da mensuração de variáveis de fácil coleta, permite estimar o rendimento de madeira serrada, bem como o volume da parte ocada das toras com maior praticidade. 
Danielli et al. - Modelagem do rendimento no desdobro de toras de

Manilkara spp. (Sapotaceae) em serraria do estado de Roraima, Brasil

O aumento do rendimento no desdobro das toras na serraria pode ser alcançado por meio da adoção de técnicas simples. Como, por exemplo, a melhoria do armazenamento de toras no pátio das serrarias. Assim como a utilização de equipamentos com maior precisão e com manutenção regular, bem como o treinamento de mão de obra e o desenvolvimento de novos produtos que possam alcançar outros mercados consumidores.

\section{REFERENCIAS BIBLIOGRÁFICAS}

ABREU, E. C. R. Modelagem para prognose precoce do volume por classe diamétrica para Eucalyptus grandis. 2000. 69 p. Dissertação (Mestrado em Ciências Florestais) - Universidade Federal de Lavras, Lavras, 2000.

ANGELINI, F.; MILONE, G. Estatística Geral. São Paulo: Atlas, 1993. 206 p.

ARAUJO, H. J. B. Aproveitamento de resíduos das indústrias serraria do Acre para fins energéticos. Rio Branco: Embrapa Acre, 2003. 38 p. (Documentos, 82)

BARROS, D. A.; MACHADO, S. A.; ACERBI JR., F. W.; SCOLFORO, J. R. S. Comportamento de modelos hipsométricos tradicionais e genéricos para plantações de Pinus oocarpa em diferentes tratamentos. Boletim de Pesquisa Florestal, Colombo, n. 45, p. 3-28, 2002.

BATISTA, D. C.; CARVALHO, A. M. Avaliação do desempenho operacional de uma serraria através de estudo do tempo, rendimento e eficiência. Scientia Forestalis, Piracicaba, n. 75, p. 31-38, 2007.

BELSLEY, O. A.; KUH, E.; WELSCH, R. E. Regression diagnostics: Identifying data and sources of collinearity. Hoboken: John Wiley \& Sons, 1980. 292 p.

BIASI, C. P.; ROCHA, M. P. Rendimento em madeira serrada e quantificação de resíduos para três espécies tropicais. Floresta, Curitiba, v. 37, n. 1, p. 95-108, 2002.

BRAND, M. A.; MUÑIZ, G. I. B.; SILVA, D. A.; KLOCK, U. Caracterização do rendimento e quantificação dos resíduos gerados em serraria através do balanço de materiais. Floresta, Curitiba, v. 32, n. 2, p. 247-259, 2002.

CAVAlLET, J.; OlIVEIRA, A. L. A.; ARRUDA, T. P. M.; ACOSTA, F. C. Rendimento em madeira de Jatobá (Hymenaea courbaril). In: SIMPÓSIO DE INICIAÇÃO CIENTÍFICA DAS CIÊNCIAS AGRÁRIAS, 2, 2010, Alta Floresta. Anais... Alta Floresta, 2010. p. 20-23.

CLEMENT, C. R.; HIGUCHI, N. A floresta amazônica e o futuro do Brasil. Ciência e Cultura, São Paulo, v. 58, n. 3, p. 44-49, 2006.

DOBNER JR., M.; HIGA, A. R.; ROCHA, M. P. Rendimento em Serraria de Toras de Pinus taeda: Sortimentos de Grandes Dimensões. Floresta e Ambiente, Seropédica, v. 19, n. 3, p. 385-392, 2012.

DRAPER, N. R.; SMITH, H. Applied regression analysis. 2.ed. New York: John Wiley \& Sons, 1966. 407 p.

DUTRA, R. I. J. P.; NASCIMENTO, S. M.; NUMAZAWA, S. Resíduos de indústria madeireira: Caracterização, consequência sobre o meio ambiente e opções de uso. Revista científica eletrônica de Engenharia Florestal, Garça, n. 5, 2005.

GERWING, J.; VIDAL, E.; VERÍSSIMO, A.; UHL, C. Rendimento no Processamento de Madeira no Estado do Pará. Belém: Imazon, 2000. 36 p. (Série Amazônia, 18)

GOMIDE, J. L. Serraria. Viçosa: Universidade Federal de Viçosa, Imprensa universitária, p. 119, 1974. 
HIGUCHI, N.; SANTOS, J.; RIBEIRO, R. J.; MINETTE, L.; BIOT, Y. Biomassa da Parte Aérea da Vegetação da Floresta Tropical Úmida de Terra-firme da Amazônia Brasileira. Acta Amazônica, Manaus, v. 28, n. 2, p. 153$166,1998$.

HIRAI, E. H.; CARVALHO, J. O. P.; PINHEIRO, K. A. O. Estrutura da população de maçaranduba (Manilkara huberi Standley) em 84 ha de floresta natural na fazenda Rio capim, Paragominas, PA. Revista de Ciências Agrárias, Belém, n. 49, p. 65-76, 2008.

IWAKIRI, S. Rendimento e condições de desdobro de 20 espécies de madeiras da Amazônia. Acta Amazônica, Manaus, v. 20 (Único), p. 271-281, 1990.

KOCK, P. Material balances and energy required for manufacture of ten wood commodities. Georgia: Forest products Research Society, 1976. 173 p.

MACHADO, S. A.; FIGUEIREDO FILHO, A. Dendrometria. 2.ed. Guarapuava: Unicentro, 2006.316 p.

MARCHESAN, R. Rendimento e qualidade de madeira serrada de três espécies tropicais. 2012.94 p. Dissertação (Mestrado) - Universidade Federal do Paraná, Curitiba, 2012.

NASCIMENTO, S. M.; DUTRA, R. I. J. P.; NUMAZAWA, S. Resíduos de indústria madeireira: caracterização, conseqüências sobre o meio ambiente e opções de uso. Holos Environment, Rio Claro, v. 6, n. 1, p. 08- 21, 2006.

PEREIRA, D.; SANTOS, D.; VEDOVETO, M.; GUIMARÃES, J.; VERÍSSIMO, A. Fatos florestais da Amazônia 2010. Belém: Imazon, 2010. 124 p.

RIBEIRO, J. Avaliação ambiental econômica da produção de madeira de espécie nativa em dois municípios na Amazônia brasileira. 2008. 42 p. Dissertação (Mestrado em Saúde Ambiental) - Setor Ciências Biológicas, Universidade de São Paulo. São Paulo, 2008.

SANTOS, J. Análise de modelos de regressão para estimar a fitomassa da floresta tropical úmida de terrafirme da Amazônia Brasileira. 1996. 121 p. Tese (Doutorado) - Universidade Federal de Viçosa, Viçosa, 1996.

SOLZA, A. N.; OliveIRA, A. D.; SCOLfORO, J. R. S.; MELlO, J. M.; CARVALHO, L. M. T. Modelagem do rendimento no desdobro de toras de eucalipto cultivado em sistema agroflorestal. Cerne, Lavras, v. 13, n. 2, p. 222-238, 2007.

SUDAM - SUPERINTENDÊNCIA DO DESENVOLVIMENTO DA AMAZÔNIA. Rendimento em serraria de trinta espécies de madeiras amazônicas. Belém. 1981. 186 p.

TONINI, H.; FERREIRA, L. M. M. Rendimento em madeira serrada de cupiúba (Goupia glabra), caferana (Erisma uncinatum) e angelim-pedra (Dinizia excelsa). Boa Vista: EMBRAPA Roraima, 2004. 6 p. (Comunicado Técnico, 7)

VALÉRIO, A. F.; WATZLAWICK, L. F; BALBINOT, R.; CALDEIRA, M. V. W.; FIGUEIREDO FILHO, A. Modelagem para a estimativa do rendimento no desdobro de toras de Araucaria angustifolia (Bertol.) Kuntze. Floresta, Curitiba, v. 39, n. 3, p. 619-628, 2009.

Recebido em 12/06/2015

Aceito para publicação em 28/01/2016

Sci. For., Piracicaba, v. 44, n. 111, p. 641-651, set. 2016 DOI: dx.doi.org/10.18671/scifor.v44n111.10 
